\title{
Diverse Precerebellar Neurons Share Similar Intrinsic Excitability
}

\author{
Kristine E. Kolkman, ${ }^{1,2}$ Lauren E. McElvain, ${ }^{1,2}$ and Sascha du Lac ${ }^{1,2,3}$ \\ ${ }^{1}$ Neurosciences Graduate Program, University of California, San Diego, La Jolla, California 92037, ${ }^{2}$ The Salk Institute for Biological Studies, La Jolla, \\ California 92037, ${ }^{3}$ Howard Hughes Medical Institute, La Jolla, California 92037
}

The cerebellum dedicates a majority of the brain's neurons to processing a wide range of sensory, motor, and cognitive signals. Stereotyped circuitry within the cerebellar cortex suggests that similar computations are performed throughout the cerebellum, but little is known about whether diverse precerebellar neurons are specialized for the nature of the information they convey. In vivo recordings indicate that firing responses to sensory or motor stimuli vary dramatically across different precerebellar nuclei, but whether this reflects diverse synaptic inputs or differentially tuned intrinsic excitability has not been determined. We targeted whole-cell patch-clamp recordings to neurons in eight precerebellar nuclei which were retrogradely labeled from different regions of the cerebellum in mice. Intrinsic physiology was compared across neurons in the medial vestibular, external cuneate, lateral reticular, prepositus hypoglossi, supragenual, Roller/intercalatus, reticularis tegmenti pontis, and pontine nuclei. Within the firing domain, precerebellar neurons were remarkably similar. Firing faithfully followed temporally modulated inputs, could be sustained at high rates, and was a linear function of input current over a wide range of inputs and firing rates. Pharmacological analyses revealed common expression of Kv3 currents, which were essential for a wide linear firing range, and of SK (small-conductance calcium-activated potassium) currents, which were essential for a wide linear input range. In contrast, membrane properties below spike threshold varied considerably within and across precerebellar nuclei, as evidenced by variability in postinhibitory rebound firing. Our findings indicate that diverse precerebellar neurons perform similar scaling computations on their inputs but may be differentially tuned to synaptic inhibition.

\section{Introduction}

The cerebellum adaptively adjusts motor and cognitive actions to meet organismal needs in the face of changes in the external and internal environment. To perform appropriately, the cerebellum requires accurate representations of intended actions and environmental conditions as well as of motor commands and their sensory consequences. This diverse information is conveyed to the cerebellum via mossy fiber axons of precerebellar neurons widely distributed in the midbrain, pons, medulla, and spinal cord.

Precerebellar mossy fiber neurons are remarkably diverse with respect to both the stimuli they respond to and their firing responses in vivo (Eccles et al., 1971; Lisberger and Fuchs, 1978; Noda, 1986; van Kan et al., 1993; Zhang et al., 1995; Cheron et al., 1996; Escudero et al., 1996; Garwicz et al., 1998; Mackie et al., 1999; Rancz et al., 2007; Bengtsson and Jorntell, 2009; Tziridis et al., 2009, 2011). For example, neurons in the external cuneate

Received June 29, 2011; revised Sept. 7, 2011; accepted Sept. 27, 2011.

Author contributions: K.E.K., L.E.M., and S.d.L. designed research; K.E.K. and L.E.M. performed research; K.E.K., L.E.M., and S.d.L. analyzed data; K.E.K. and S.d.L. wrote the paper.

This work was supported by NIH Grant EY11027, the HHMI, and an NIH T32 Systems and Integrative Neurobiology Grant to K.E.K. We thank Alexandra Sakatos, Brian Zingg, and Michael Fuentes for excellent technical support.

The authors declare no competing financial interests.

Correspondence should be addressed to Sascha du Lac, Howard Hughes Medical Institute, The Salk Institute for

Biological Studies, Systems Neurobiology Laboratories, 10010 North Torrey Pines Road, La Jolla, CA 92037. E-mail: sascha@salk.edu.

DOI:10.1523/JNEUROSCI.3314-11.2011

Copyright $\odot 2011$ the authors $\quad 0270-6474 / 11 / 3116665-10 \$ 15.00 / 0$ nucleus fire transient, high-frequency bursts in response to direct sensory (cutaneous) stimulation (Bengtsson and Jorntell, 2009), while those in the prepositus hypoglossi exhibit graded modulations in firing rate that are proportional to eye position (Escudero et al., 1996). Neurons in pontine nuclei are driven by complex cortical signals and exhibit a wide range of firing responses even during simple sensory-motor tasks (Tziridis et al., 2009, 2011).

This diversity of firing responses raises the question of whether functionally distinct precerebellar neurons are intrinsically tuned for their specific inputs. Are some precerebellar neurons tuned as event detectors and built to burst, while others are specialized for graded signaling over a wide input range? Although the literature contains several reports of intrinsic firing properties in specific populations of precerebellar neurons (Eide et al., 1969; Kitai et al., 1976; Schwarz et al., 1997; Mock et al., 2002), discrepancies in recording conditions preclude cogent comparisons across nuclei. To determine whether intrinsic firing properties vary across functionally distinct cell types, we probed intrinsic excitability of neurons recorded in eight different precerebellar nuclei in brainstem slices.

\section{Materials and Methods}

Animals. All protocols were approved by the Animal Care and Use Committee at the Salk Institute in accordance with National Institute of Health guidelines. Neurons were imaged and recorded in wild-type and transgenic mice of either gender [GlyT2 (Zeilhofer et al., 2005), GIN (Oliva et al., 2000), YFP-16 (Feng et al., 2000), L7-GFP (Sekirnjak et al., 2003)], which were backcrossed (at least five generations) to the C57BL/6 background. 
Tracer injection. Angle Two stereotaxic apparatus and software (myNeuroLab.com) were used to target injections to the flocculus and vermis/hemisphere; these regions of the cerebellum each receive mossy fiber inputs from several different precerebellar nuclei. For injections in adult mice [postnatal day 70 (P70) or older], the flocculus was targeted $3.32 \mathrm{~mm}$ lateral, $5.68 \mathrm{~mm}$ posterior, and $4.81 \mathrm{~mm}$ ventral to bregma at an angle of $30.69^{\circ}$ on the mediolateral axis. For flocculus injections in young mice (age P16-P23), the flocculus was targeted at $3.32 \mathrm{~mm}$ lateral, 5.08 mM posterior, and $4.81 \mathrm{~mm}$ ventral to bregma at a $25^{\circ}$ mediolateral angle.

Texas Red dextran (molecular weight, 10,000) was used as the neuronal tracer. Injections were made using either dextran crystals or a nearsaturated solution of dextran in deionized water. Brain slices were made 2-4 d after surgery for electrophysiological recordings in young mice or 3-7 d later for histological analysis in adult mice.

Fixed tissue preparation. Animals were perfused transcardially with PBS and subsequently with $4 \%$ paraformaldehyde in PBS. The brains were removed and placed in $4 \%$ paraformaldehyde in PBS at room temperature for $30 \mathrm{~min}$. The brains were then transferred to $4^{\circ} \mathrm{C}$ and placed in $30 \%$ sucrose in PBS until the brains sank $(\sim 24 \mathrm{~h})$. Thin coronal sections $(40 \mu \mathrm{m})$ were sliced with a freezing microtome (Microm) through the brainstem and pons. Slices were wet mounted and coverslipped with $2.5 \%$ DABCO (1,4 diazabicyclo-[2.2.2] octane).

Cerebellum injection sites and retrogradely labeled precerebellar neurons were imaged using an Olympus BX61 fluorescent microscope equipped with a Hamamatsu CCD camera (C4742-95). A calcium crimson filter (Olympus) was used to image Texas Red dextran fluorescence. The cerebellum and brainstem were imaged with bright-field imaging. Images were collected digitally using ImageJ software; then, brightness and contrast were adjusted and the image histograms stretched in Adobe Photoshop. The areas surrounding the imaged tissue were artificially darkened to increase signal-tonoise contrast. The slices imaged for Figure 1 were imaged under dark-field illumination to confirm the nuclear borders.

Slice preparation for electrophysiology. Before brain tissue was harvested, mice (P18-P25) were deeply anesthetized with Nembutal and then decapitated. Their hindbrains were removed and dissected in icecold carbogenated $\left(95 \% \mathrm{O}_{2} / 5 \% \mathrm{CO}_{2}\right)$ artificial CSF (ACSF) containing the following (in mM): $124 \mathrm{NaCl}, 5 \mathrm{KCl}, 1.3 \mathrm{MgSO}_{4}, 26 \mathrm{NaHCO}, 2.5$ $\mathrm{CaCl} 2,1 \mathrm{NaH}_{2} \mathrm{PO}_{4}$, and 11 glucose. When carbogenated, ACSF had a $\mathrm{pH}$ of 7.4 and an osmolarity of $300 \mathrm{mOsm}$. Coronal slices ( $250 \mu \mathrm{M}$ thick) of the brainstem and pons were cut on either a Leica VT1000S or DSK$1500 \mathrm{E}$ vibratome. Slices were incubated in a holding chamber containing carbogenated ACSF at $34^{\circ} \mathrm{C}$ for $45 \mathrm{~min}$ and then stored in carbogenated room-temperature ACSF before recording to prolong slice longevity.

Electrophysiological recording. Recordings were made in a submersion chamber with constant perfusion of carbogenated ASCF at $34^{\circ} \mathrm{C}$. Picrotoxin $(100 \mu \mathrm{M})$ and strychnine $(1 \mu \mathrm{M})$ were added to the ACSF for all recordings to block GABAergic and glycinergic transmission, respectively. Fluorescent cells were visualized using Olympus (U-LH100HG) illumination and a calcium crimson filter (for visualizing Texas Red). They were targeted for whole-cell patch-clamp recording using a DageMTI or VE1000 camera mounted on an Olympus BX51WI infrared differential contrast microscope under $40 \times$ magnification.

Recordings were performed with an AxoClamp 2B or a MultiClamp 700B amplifier in current-clamp mode. Micropipettes (4-7 M $\Omega$ ) were made from borosilicate glass (inner diameter, $0.86 \mathrm{~mm}$; outer diameter, $1.50 \mathrm{~mm}$ ) using a Sutter Instruments P-97 puller. The solution filling the electrodes contained the following (in mM): 140 K gluconate, 10 HEPES, $8 \mathrm{NaCl}$, 0.1 EGTA, $2 \mathrm{MgATP}$, and 0.3 Na2GTP, adjusted to pH 7.3-7.5 and $280-285 \mathrm{mOsm}$. Data were filtered at $10 \mathrm{kHz}$ and digitized at $40 \mathrm{kHz}$ with an ITC-18 interface (Instrutech). Data acquisition and analysis was performed using code written in-house in Igor 6. A liquid junction potential of $14.5 \mathrm{mV}$, calculated with JPCalc, was subtracted from all membrane potential measurements.

Analysis of electrophysiological parameters. Action potential profiles were acquired for $1-5 \mathrm{~s}$ at a rate of $8-12$ spikes per second and then averaged after aligning their peaks. Action potential threshold was defined as the membrane potential where the derivative of the voltage trace reached $10 \mathrm{~V} / \mathrm{s}$. The width of the action potentials was determined at threshold, and the half-width was defined as the width halfway between threshold and peak. Afterhyperpolarization (AHP) was determined as the difference between threshold and the most hyperpolarized membrane potential between spikes. Neurons were excluded from the study if their action potential height was $<45 \mathrm{mV}$ and they could not fire faster than 50 spikes per second in response to depolarization; such neurons were rarely encountered in healthy slices and had unstable membrane potentials indicative of poor recording quality.

To measure excitability above threshold, the current-to-firing rate relationship was determined by applying $1 \mathrm{~s}$ depolarizing current steps of increasing magnitude until the neuron exhibited depolarization block and could not fire throughout the step and then measuring the average evoked firing rate. The gain of the relationship was defined as the best-fit line of the current versus mean firing rate curve. As with medial vestibular nucleus (MVN) neurons (Bagnall et al., 2007), firing responses of precerebellar neurons tended to exhibit distinct slopes above and below mean firing rates of 80 spikes per second. The maximum firing rate was defined as the mean firing rate evoked during the largest depolarization that the neuron could maintain firing throughout the $1 \mathrm{~s}$ input; larger inputs produced depolarization block of firing via accumulated inactivation of Na channels (Gittis et al., 2010).

Input resistance was measured by hyperpolarizing the neurons to -75 $\mathrm{mV}$ and averaging the change in membrane potential evoked by six repetitions of small hyperpolarizing current steps, $1 \mathrm{~s}$ in duration.

Adaptation was measured in response to $1 \mathrm{~s}$ depolarizing inputs that evoked steady-state firing rates of $\sim 150$ spikes per second. Firing rate adaptation was defined as the ratio of the firing rate over the first and last $100 \mathrm{~ms}$ of the step after excluding the initial $50 \mathrm{~ms}$ (which depended sensitively on initial membrane potential and depolarization amplitude) from the analysis (Sekirnjak and du Lac, 2002). Postinhibitory rebound firing was measured by applying DC current to maintain neuronal firing at 10 spikes per second. One second steps that hyperpolarized the neurons by $30 \mathrm{mV}$ were applied; rebound firing rate was determined as the peak difference in firing rate before and after the hyperpolarizing step.

To measure the firing rate response to sinusoidally modulating current, precerebellar neurons were depolarized to fire at 30 spikes per second. Sinusoidally modulating current was delivered at $0.25,0.5,1,2$, and $4 \mathrm{~Hz}$ with an amplitude producing modulation of $\pm 10-15$ spikes. The sinusoidal firing rate responses were fit using sinusoidal functions $f(t)=$ $A+B \sin (2 \pi f t+C)$. The frequency $(f)$ was set to the input frequency, and $A, B$, and $C$ were chosen to minimize the mean SE. Comparison of the fit sinusoid to the input sinusoid was used to determine gain and phase responses of the neurons.

Statistical analysis. Physiological parameters from the eight precerebellar nuclei recorded were compared using the Kruskal-Wallis nonparametric multiple group comparison followed by the Dunn's post hoc test (if $p<0.05$ ) performed in GraphPad Prism (version 4.0) software. Data from the eight nuclei were grouped together for pharmacological experiments. Data before and after pharmacological manipulation were compared using the paired Wilcoxon rank sum nonparametric test in Kaleidagraph (version 4.03). Values are reported as the mean \pm SD unless indicated otherwise.

\section{Results}

\section{Locations of diverse precerebellar neurons}

To identify precerebellar neurons in vitro, we made dextran tracer injections in the cerebellum. Injections targeting the flocculus (Fig. $1 A$ ), vermis and hemispheres (Fig. $1 F$ ) were confirmed by retrograde neuronal labeling in the contralateral inferior olive (Fig. $1 B, G)$. Flocculus injections also resulted in retrogradely labeled neurons (Fig. $1 C-E$ ) in the MVN, nucleus prepositus hypoglossi (NPH), supragenual nucleus, the nucleus intercalatus/nucleus of Roller, nucleus reticularis tegmenti pontis (NRTP), and lateral reticular nucleus (LRN). Vermis and hemisphere injections resulted in retrogradely labeled in the LRN, pontine nucleus, NRTP, nucleus of Roller/intercalatus, and external cuneate nucleus (Fig. $1 \mathrm{H}-\mathrm{J}$ ).

Neurons in eight brainstem nuclei that project mossy fiber axons to the cerebellum were targeted for whole-cell patch re- 


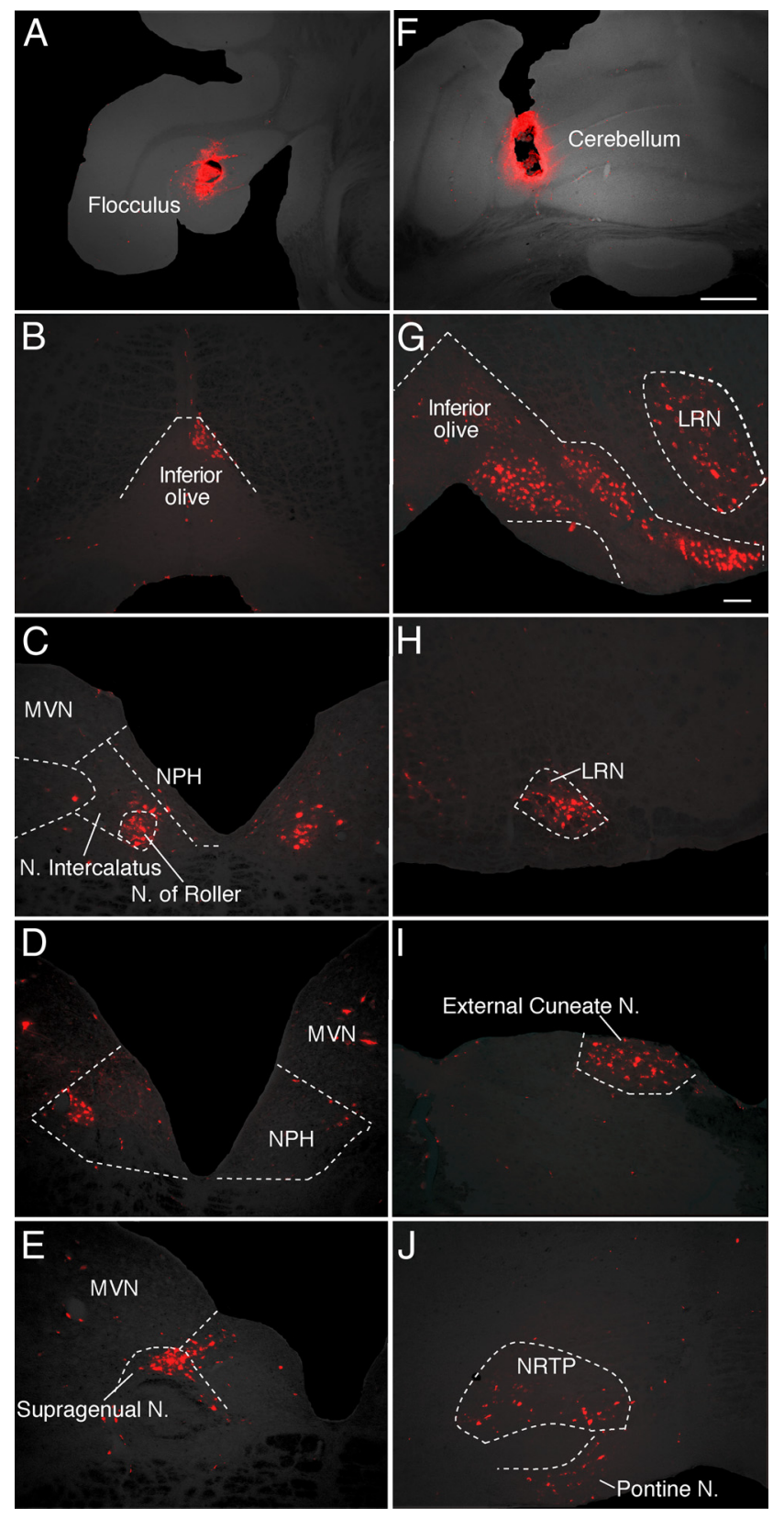

Figure 1. Identification of precerebellar neurons in pons and medulla via retrograde labeling of red fluorescent dextrans. $A$, Tracer injection site (red) in the floccular lobe of the cerebellum is shown in a coronal section $\sim 5.6 \mathrm{~mm}$ caudal to bregma. Retrogradely labeled neurons from this injection are shown in coronal sections in $\boldsymbol{B}-\boldsymbol{E}$. $\boldsymbol{B}$, Section through the caudal medulla, $\sim 7.75$ $\mathrm{mm}$ caudal to bregma, showing neurons in the inferior olive (dorsal cap) that were retrogradely labeled from the cerebellar flocculus. $\mathbf{C}-\boldsymbol{E}$, Retrogradely labeled neurons in the MVN, NPH, nucleus of Roller/nucleus intercalatus, and supragenual nucleus in medullary sections 6.96, 6.5, and $5.8 \mathrm{~mm}$ caudal to bregma, respectively. $\boldsymbol{F}$, A second example of a cerebellar injection, $\sim 6.25 \mathrm{~mm}$ caudal to bregma, which resulted in the pattern of retrograde labeling show in $\mathbf{G}-\mathbf{J}$. $G$, Retrogradely labeled neurons in several portions of the inferior olive, as well as in the LRN, are shown in this medullary section $\sim 7.56 \mathrm{~mm}$ caudal to bregma. $\boldsymbol{H}$, Retrogradely labeled neurons in the LRN, $\sim 7.76 \mathrm{~mm}$ caudal to bregma. I, Retrogradely labeled neurons in the external cuneate, adjacent to the fourth ventricle in this section, $\sim 7.35 \mathrm{~mm}$ caudal to bregma. J, Retrogradely labeled neurons in the NRTP and the pontine nuclei in a section $\sim 4.25 \mathrm{~mm}$ caudal to bregma. Scale bars: (in $\boldsymbol{F}) \boldsymbol{A}, \boldsymbol{F}, 500 \mu \mathrm{m}$; (in $\boldsymbol{G}) \boldsymbol{B}-\boldsymbol{E}, \boldsymbol{H}-\boldsymbol{J}, 100 \mu \mathrm{m}$.

cordings in brainstem slices. These nuclei were selected to represent the diversity of sensory, motor, and cortical signals entering the cerebellum. The external cuneate primarily processes sensory information, with the somatosensory system supplying the dom-
Table 1. Resting properties of precerebellar neurons recorded in eight different nuclei

\begin{tabular}{llllr}
\hline Nucleus & $n$ & \# firing spont. & Spont. FR \pm SE (spikes/s) & $V_{\text {rest }} \pm$ SE $(\mathrm{mV})$ \\
\hline External cuneate & 24 & 5 & $22 \pm 8$ & $-75.6 \pm 1.2$ \\
LRN & 13 & 4 & $8 \pm 2$ & $-66.8 \pm 1.6$ \\
MVN & 40 & 21 & $13 \pm 2$ & $-71.4 \pm 1.1$ \\
NPH & 21 & 6 & $11 \pm 7$ & $-74 \pm 1.3$ \\
NRTP & 30 & 1 & 9 & $-76 \pm 1.0$ \\
Pontine & 20 & 0 & NA & $-78 \pm 1.6$ \\
Roller & 27 & 8 & $16 \pm 4$ & $-72 \pm 1.7$ \\
Supragenual & 23 & 4 & $12 \pm 2$ & $-73 \pm 1.3$ \\
\hline
\end{tabular}

$V_{\text {rest }}$ indicates resting membrane potential of neurons that did not fire spontaneously. Spont., Spontaneously; NA, not applicable; $F R$, firing rate.

inant inputs. In contrast, the NRTP primarily processes motor information, receiving efference copies of eye movement commands. The LRN integrates proprioceptive and autonomic signals with descending motor commands. The MVN and NPH process a combination of sensory and motor information: head motion sensory signals from the inner ear and efference copies of eye movement commands. The supragenual and Roller nuclei process brainstem and cerebellar signals related to gaze. Finally, the pontine nuclei convey highly processed signals from widespread regions of the cerebral cortex to the cerebellum.

Several precerebellar nuclei project axons bilaterally to the cerebellum, including the MVN, NPH, supragenual nucleus, and nucleus of Roller/intercalatus. The intrinsic physiological properties of neurons retrogradely labeled ipsilaterally versus contralaterally to the injection site in the cerebellar cortex were compared for MVN, NPH, nucleus of Roller/intercalatus, and supragenual neurons projecting to the flocculus. Ipsilaterally and contralaterally projecting neurons in each nucleus were indistinguishable with respect to membrane and firing properties and thus were grouped.

\section{Action potential waveforms are similar across precerebellar neurons}

Some retrogradely labeled neurons in most precerebellar nuclei fired spontaneously in whole-cell patch recordings, while other neurons recorded in the same brain slice preparation and nuclei were silent at rest. The highest proportion of neurons firing spontaneously was found in the MVN (52\%), while about 20-35\% of neurons fired spontaneously in the LRN, NPH, Roller, external cuneate, and supragenual nuclei (Table 1). In contrast, only one neuron (of 30) in the NRTP fired spontaneously, and all pontine neurons were silent in the absence of exogenous depolarization (Table 1). Spontaneous firing rates ranged from 2 to 43 spikes per second and were not significantly different across nuclei.

Despite differences within and across nuclei in resting activity, the action potential profiles of precerebellar neurons were remarkably similar. Figure 2 shows representative action potentials of precerebellar neurons from each of the eight nuclei targeted for recording. To standardize comparison across neurons, action potentials were measured at firing rates of 10 spikes per second, maintained as necessary by intracellular current injection. As evidenced by each of the eight examplar neurons, the action potential comprises both a rapidly rising and a repolarizing phase and is followed by a biphasic AHP consisting of an early, fast phase and a second, slower phase. The width of the action potential (measured at half-height; see Materials and Methods) averaged 0.35 $\mathrm{ms}$, and the peak amplitude of the AHP averaged $17.6 \mathrm{mV}$. These parameters varied within nuclei, but their population values were statistically indistinguishable across nuclei. The similarity of ac- 
tion potential waveforms across eight precerebellar nuclei suggests similarities in the underlying ionic currents.

\section{Firing responses to depolarizing current}

Precerebellar neurons recorded in vivo exhibit a range of firing properties. To investigate whether these responses reflect differences in intrinsic excitability across precerebellar nuclei, we assessed firing responses to intracellular depolarization. All precerebellar neurons fired continuously in response to $1 \mathrm{~s}$ of depolarization. Responses to a range of input current amplitudes, plotted as instantaneous firing rate versus time, are shown for a typical precerebellar neuron (from the pontine nucleus) in Figure 3A. Following the stimulus onset, the neuron exhibited a rapid increase in firing rate that was proportional to the input amplitude and adapted modestly over the course of $1 \mathrm{~s}$. In response to inputs $>3.5 \mathrm{nA}$, the neuron exhibited depolarization block and was unable to fire continuously throughout the $1 \mathrm{~s}$ stimulus. Figure $3 B$ plots the mean evoked firing rate as a function of the input current and demonstrates a typical feature of precerebellar neuronal inputoutput properties; firing rates are a bilinear function of input currents, exhibiting a change in gain (slope) at firing rates of 80 spikes per second. For the neuron shown in Figure $3, A$ and $B$, the gain (evoked firing rate divided by input current) of the firing responses below 80 spikes per second was 79.9 spikes/s/nA, the gain of firing responses above 80 spikes per second was 50.5 spikes $/ \mathrm{s} / \mathrm{nA}$, and the maximum firing rate (see Materials and Methods) was 183 spikes per second.

Firing responses to current steps ranged widely but were qualitatively similar across precerebellar neurons recorded in different nuclei. Maximum firing rates did not differ significantly across nuclei; on average, the maximum firing rate was 170 spikes per second (Fig. 3C). Almost all neurons exhibited two ranges of linear firing responses to inputs, such that the ratio of gains derived for values fit below versus above 80 spikes per second averaged between 1.5 and 1.9 (Fig. 3F). Within both ranges, firing responses were almost perfectly linear functions of input current amplitude, as evidenced by correlation coefficients that averaged 0.99 (Fig. 3D). Gains in both ranges varied significantly between precerebellar nuclei ( $p=0.00003)$. MVN and LRN neurons exhibited the highest and lowest gains, respectively, averaging 164 and 99 spikes/s per nA when measured below 80 spikes per second. The extent of firing rate adaptation also varied across precerebellar neurons within and across nuclei (Fig. $3 G$; $p=0.025$ ), but statistical post hoc tests failed to show individual differences. Differences in excitability were not reflected in analyses of input resistance measured below spike threshold; input resistances, which were typically between 100 and $200 \mathrm{M} \Omega$, did not vary significantly across nuclei (Fig. $3 H$ ).

\section{Firing responses to temporally modulated inputs}

To examine firing responses to temporally modulated inputs, we alternately depolarized and hyperpolarized neurons using sinusoidal current injection. To standardize the comparisons and to prevent firing rates from rectifying, temporally modulated current was delivered to neurons firing at baseline rates between 25 and 30 spikes per second (evoked by DC current injection), and sinusoidal current was constrained to produce peak-to-peak modulations that averaged 20 spikes per second. Firing responses of a typical precerebellar neuron, recorded in the nucleus of Roller/intercalatus, to sinusoidally modulated current are shown in Figure 4A. This and all other precerebellar neurons exhibited remarkably faithful modulation of firing rate in response to temporally modulated inputs, resulting in excellent fits of sine waves to instantaneous firing rate (Fig. $4 \mathrm{~A}$, middle).

Firing rate responses to sinusoidal inputs ranging from 0.25 to $4 \mathrm{~Hz}$ are plotted as a function of input frequency in Figure $4 B$. Each data point represents the population average and SE of the population responses for each of eight different precerebellar nuclei. Firing responses to sine waves were qualitatively identical across neurons and nuclei. Response gains increased slightly and systematically as input frequency increased from 0.25 to $4 \mathrm{~Hz}$ (Fig. 4B). Firing responses were nearly in phase with input currents, exhibiting a slight phase lead at the lowest input frequencies and a slight phase lag at the highest input frequencies (Fig. 4C).

As with responses to depolarizing steps, precerebellar neurons from distinct nuclei exhibited quantitative differences in sinusoi- 
A

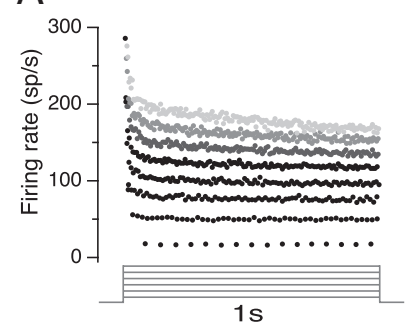

C

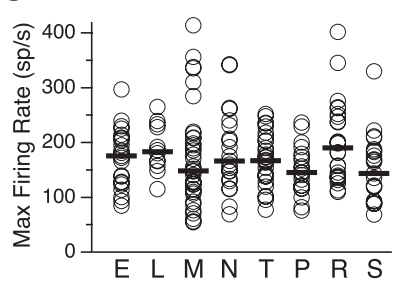

E

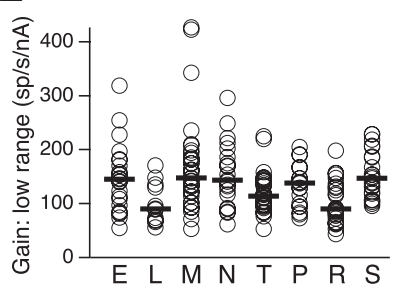

G

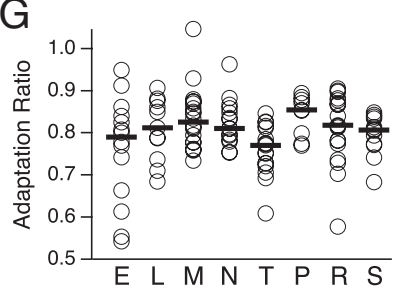

B

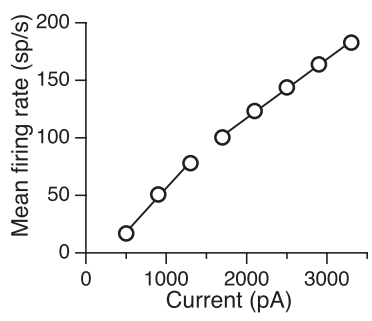

D

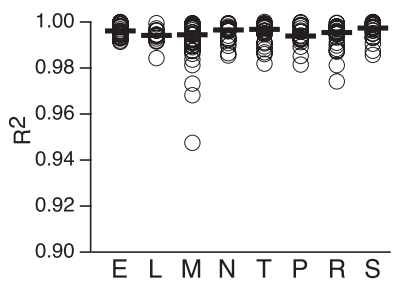

F
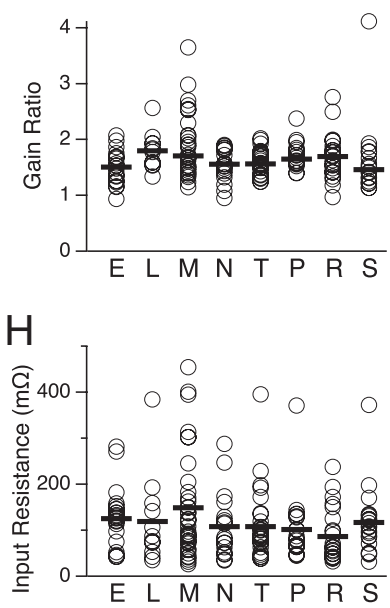

Figure 3. Precerebellar neurons transform input current into firing rate linearly. $\boldsymbol{A}$, Instantaneous firing rate versus time for a retrogradely labeled neuron recorded in the NRTP in response to $1 \mathrm{~s}$ depolarizing inputs of increasing magnitude. $\boldsymbol{B}$, The relationship between current and mean firing rate across $1 \mathrm{~s}$ depolarizing steps for the neuron shown in $\boldsymbol{A}$. Lines represent best linear fits to firing rate responses below and above 80 spikes per second. $C$, Maximum firing rates for all recorded precerebellar neurons. $D$, Linear correlation coefficient $\left(R^{2}\right)$ for current-tofiring rate relationship below 80 spikes per second. $\boldsymbol{E}$, Gain of the current-to-firing rate relationship below 80 spikes per second. Significant differences of $p<0.05$ were found for $L$ versus $M$ or $S$, and $R$ versus $M, N$, or $S$. $F$, Ratio of gain for the current-to-firing rate relationship under and over 80 spikes per second. $\boldsymbol{F}$, Ratio of gain for the current-to-firing rate relationship below and above 80 spikes per second. $\mathbf{G}$, Adaptation ratio indicates the ratio of the firing rate at the end versus the start of the $1 \mathrm{~s}$ current step measured at steady-state firing rates of 150 spikes per second. $\boldsymbol{H}$, Input resistance measured below spike threshold. E, External cuneate; L, lateral reticular nucleus; $M$, medial vestibular nucleus; $N$, nucleus prepositus hypoglossi; $T$, nucleus reticularis tegmenti pontis; $P$, pontine nucleus; $R$, nucleus of Roller/nucleus intercalatus; $S$, supragenual nucleus. Open symbols are individual data points. Black horizontal bars represent the median value for each nucleus.

dal gain (Fig. $4 B ; p=0.00004$ ). MVN neurons had significantly higher gains $(p<0.05)$ across all frequencies than did pontine, nucleus of Roller/intercalatus, and NRTP neurons, which exhibited the lowest gains. In contrast, phase responses were quantitatively identical across frequencies for all precerebellar nuclei except at the lowest frequencies, where the MVN had a slight but significant phase lead relative to the nucleus of Roller/intercalatus $(0.25$ and $0.5 \mathrm{~Hz}, p<0.05)$ and the $\operatorname{LRN}(0.25 \mathrm{~Hz}, p<0.05)$. Together, these results indicate that the firing properties of
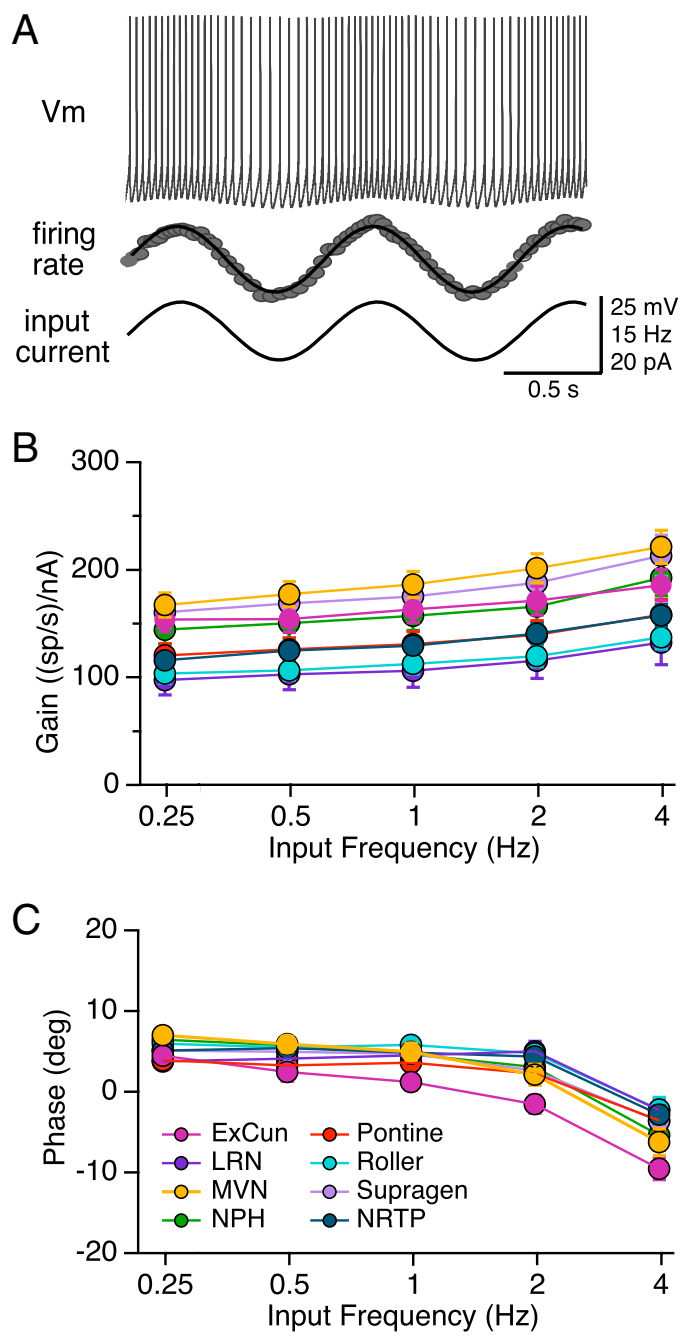

Figure 4. Mossy fiber neuron firing rate modulation in response to sinusoidal inputs. $\boldsymbol{A}$, Top trace, Voltage recording of a mossy fiber neuron firing during sinusoidal modulation. Middle trace, Firing rate of the neuron. Bottom trace, Sinusoidal current input to the soma. $\boldsymbol{B}, \boldsymbol{C}$, Bode plots summary of gain $(\boldsymbol{B})$ and phase $(\boldsymbol{C})$ in mossy fiber neurons. Symbols represent the average response of neurons in the eight precerebellar nuclei. Error bars indicate SEM. ExCun, External cuneate; Roller, nucleus of Roller/nucleus intercalatus; Supragen, supragenual nucleus.

precerebellar neurons are qualitatively similar but exhibit some quantitative differences.

The intrinsic excitability of neurons identified by neurotransmitter expression, synaptic input, and/or projection pattern have been examined extensively in the dorsal brainstem (Sekirnjak et al., 2003; Takazawa et al., 2004; Sekirnjak and du Lac, 2006; Bagnall et al., 2007; Shino et al., 2008; Kolkman et al., 2011; Shin et al., 2011). Despite similarities in the ability to fire in a sustained, linear fashion, firing properties of glutamatergic neurons that do not project to the cerebellum are quantitatively different from neighboring precerebellar neurons. Neurons in the MVN that project rostrally to the oculomotor nucleus (Sekirnjak and du Lac, 2006) or caudally to the reticular formation (Kolkman et al., 2011) adapt less and can fire much faster than the population of precerebellar neurons reported here (Table 2). As a population, precerebellar neurons had significantly lower gains than did neurons that project to the oculomotor nucleus or reticular formation (Table 2). These results indicate that precerebellar neurons in different nuclei share intrinsic properties and differ significantly from other brainstem neurons that do not convey information to the cerebellum. 
Table 2. Comparisons of intrinsic excitability in neurons projecting to the cerebellum, oculomotor nucleus, and reticular formation

\begin{tabular}{|c|c|c|c|c|}
\hline & Precerebellar median (1st and 3rd quartiles), $n$ & OMN-projecting median (1st and 3rd quartiles), $n$ & Reticular-projecting median (1st and 3rd quartiles), $n$ & $p$ value \\
\hline Max firing rate & $164(123,237), 170$ & $316(248,405), 37$ & $244(154,336), 33$ & $\begin{array}{r}<0.0001 \\
0.0016\end{array}$ \\
\hline Adaptation index & $0.81(0.77,0.86), 150$ & $0.88(0.86,0.9), 37$ & $0.86(0.82,0.88), 30$ & $\begin{array}{r}<0.0001 \\
0.0022\end{array}$ \\
\hline Gain & $83(56,103), 150$ & $188(149,221), 37$ & $123(101,137), 30$ & $\begin{array}{l}<0.0001 \\
<0.0001\end{array}$ \\
\hline
\end{tabular}

Values are the median and 1st and 3rd quartiles. Gain values are reported for evoked firing rates of $>80$ spikes per second. Statistical significance was determined with a nonparametric Wilcoxon sign rank test and is reported for precerebellar versus oculomotor nucleus (OMN) and reticular projecting neurons, respectively. Data for neurons projecting to the OMN and reticular formation were from Sekirnjak and du Lac (2006) and Kolkman et al (2011), respectively.

\section{Slow-conductance calcium-activated potassium channels decrease firing response gain and maximum firing rates}

The data presented so far indicate that precerebellar neurons are similar in their action potential waveforms, their ability to sustain high firing rates, and their remarkably faithful and linear firing responses to input currents, suggesting that they share underlying conductances. Which ion channels are required to generate linear firing responses over a wide range of inputs and firing rates?

Small-conductance calcium-activated potassium (SK) currents influence excitability in many types of neurons (Sah, 1996), including neurons in the MVN (Smith et al., 2002). We examined the role of SK currents in precerebellar neurons by assessing the effects of pharmacological manipulation with the specific blocker apamin. Subsaturating concentrations of apamin $(50 \mathrm{~nm})$ were used to preclude high-frequency bursting. Partial blockade of SK currents had no effect on the rising and falling phases of the action potential but diminished both the fast and slow components of the AHP, as evidenced in a representative precerebellar neurons recorded in the LRN in Figure $5 \mathrm{~A}$. The decrease in AHP amplitude was consistent across all precerebellar neurons, with an average decrease of $6 \mathrm{mV}$ (Fig. 5D; $p<0.01)$. Firing responses to input current steps increased dramatically in the presence of apamin (Fig. $5 B$ ), resulting in a large increase in gain across the entire firing range (Fig. $5 C$ ). Partial blockade of SK channels increased gain twofold to fourfold across all precerebellar neurons tested (Fig. $5 F ; n=9$; gain measured below 80 spikes per second; $p<0.05)$. The increase in gain was accompanied by a significant increase in adaptation (Fig. $5 B, E$; $p<0.05)$. Interestingly, blockade of SK channels resulted in a $>50 \%$ increase in maximum firing rates and a decrease in the range of input currents over which neurons could sustain firing (Fig. $5 G ; p<0.01$ and $p<0.05$, respectively). Together, these results indicate that SK currents active during the interspike interval are crucial for the typical firing responses of precerebellar neurons and that they act to reduce excitability and increase the input range over which neurons fire proportionately with their inputs.

Big-conductance calcium-activated potassium channels decrease firing response gain

Big-conductance calcium-activated potassium (BK) currents influence excitability in many cell types (Sah, 1996; Smith et al.,
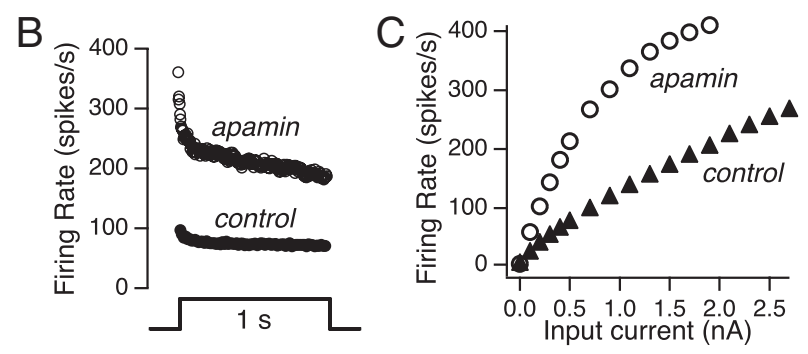

E

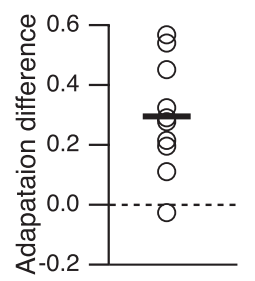

$\mathrm{F}$

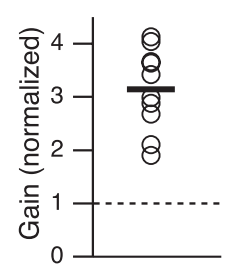

G

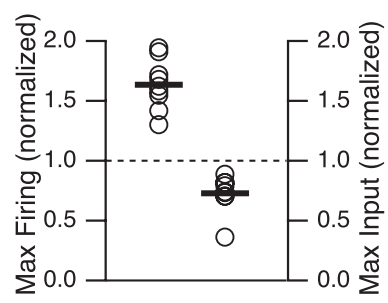

Figure 5. SK channels constrain the gain and maximum firing rate of mossy fiber neurons. $\boldsymbol{A}$, Example of an action potential 作

2002) and are targets for activity-dependent plasticity in MVN neurons (Nelson et al., 2003, 2005). We examined the role of BK currents in intrinsic excitability of precerebellar neurons by assessing the effects of the BK channel blocker paxilline $(1 \mu \mathrm{M})$. Similar to the results from blocking SK channels, blockade of BK channels with paxilline reduced the magnitude of the AHP, although the average magnitude of the decrease was half of that with SK blockade (Fig. 6A; $p<0.01$ ). Firing response gains for firing rates $<80$ spikes per second were increased slightly in paxilline (on average by $19 \%$; $p<0.05 ; n=10$; Fig. $6 D$ ), but this effect was significantly less dramatic than the doubling to quadrupling of gain in apamin (compare Fig. 5F). Paxilline did not have a significant effect on input current range (Fig. $6 E$ ) or maximum firing rate (Fig. $6 F$ ). These results indicate that BK currents have a modest effect on precerebellar neuronal gain but are not essential for their canonical firing properties.

\section{Kv3 channels enable high firing rates}

Kv3 currents are critical for rapid neuronal firing (Rudy and McBain, 2001). We used tetraethylammonium (TEA) to examine the contributions of Kv3 currents to firing in precerebellar neurons. At low concentrations ( $1 \mathrm{~mm})$, TEA blocks Kv3 and BK channels as well as dendrotoxin-sensitive channels, which do not contribute to excitability in MVN neurons (Gittis and du Lac, 2007). To examine the role of Kv3 currents specifically, we first 
A

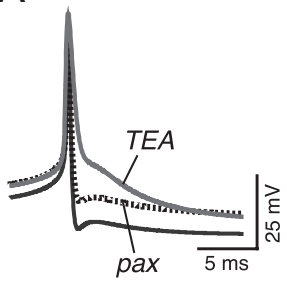

$\mathrm{B}$

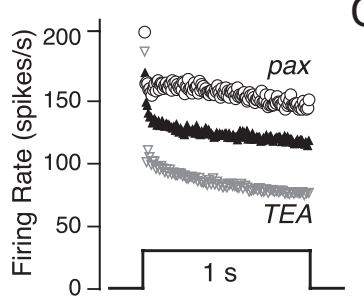

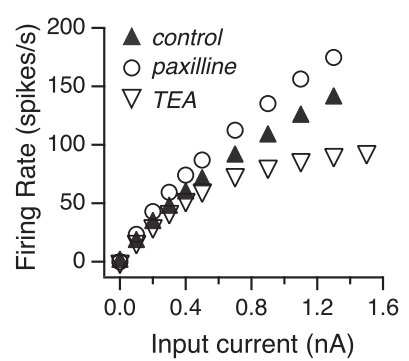
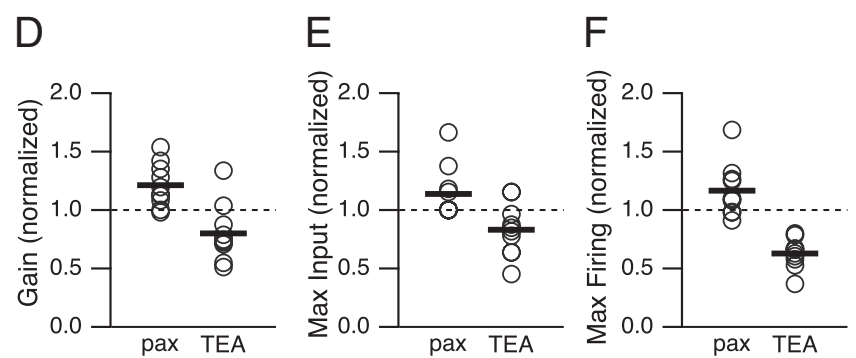

Figure 6. BK and Kv3 currents contribute to gain and maximum firing rates of mossy fiber neurons. $A$, Example of an action potential before and after application of paxilline $(I \mu \mathrm{M})$ and paxilline plus TEA (I $\mu \mathrm{M}$ and $1 \mathrm{mM}$, respectively) in a precerebellar neuron recorded in the nucleus of Roller. $\boldsymbol{B}$, Instantaneous firing rate versus time during a $1 \mathrm{~s}$ depolarizing current step before and after application of paxilline and paxilline plus TEA.C, Relationship between current and mean firing rate over $1 \mathrm{~s}$ depolarizing steps before and after paxilline and paxilline/TEA application. $\boldsymbol{D}-\boldsymbol{F}$, Change in gain below 80 spikes per second (D), maximum input $(\boldsymbol{E})$, and maximum firing rate $(\boldsymbol{F})$ normalized to the control values. $\boldsymbol{G}$, Change in AHP amplitude after paxilline and paxilline/TEA exposure. $\boldsymbol{D}-\mathbf{G}$ show results of paxilline and TEA on precerebellar neurons recorded in each of the eight precerebellar nuclei analyzed in this manuscript (2 neurons each from NPH and the nucleus of Roller).

blocked BK currents with paxilline and then applied 1 mM TEA. Blockade of Kv3 currents had a dramatic effect on action potential repolarization and abolished the early phase of the AHP $(p<$ 0.01 ) but had no effect on the late phase of the AHP (Fig. $6 A$ ). Firing response gain was reduced slightly in the presence of $1 \mathrm{mM}$ TEA at low firing rates $(p<0.01)$ but reduced dramatically at higher firing rates $(p<0.05$; Fig. $6 C)$. As a consequence, the maximum firing rate decreased significantly $(p<0.01$; Fig. $6 C)$. Across precerebellar neurons, blockade of Kv3 currents produced an average decrease in maximum firing rate of 56 spikes per second, a decrease to $62 \%$ of the control value. In contrast, TEA had a small but significant effect on the input current range $(p<0.05$; Fig. $6 E$ ). These results indicate that Kv3 currents are essential for high firing rates attained by precerebellar neurons but have little influence on firing responses below 80 spikes per second.

\section{Postinhibitory rebound firing}

The results presented thus far indicate that neurons in different precerebellar nuclei have similar firing responses to a wide range of inputs. At rest, however, some precerebellar neurons fire spontaneously while others are silent, indicating that ionic conductances near spike threshold may differ across precerebellar neurons and nuclei. To further explore such differences, we examined responses to stimuli that hyperpolarized neurons below spike threshold. To standardize the baseline conditions and to compare with previous literature in brainstem neurons (Sekirnjak and du Lac, 2002), hyperpolarizing currents steps that reduced the average membrane potential by $30 \mathrm{mV}$ were applied to neurons firing at 10 spikes per second (maintained as necessary with intracellular DC current). In most neurons, including the examples shown in Figures 7, $A 1$ and $B 1$, hyperpolarization produced an initial decrease in membrane potential followed by a depolarizing sag in membrane hyperpolarization that is characteristic of the hyperpolarization-activated cationic conductance

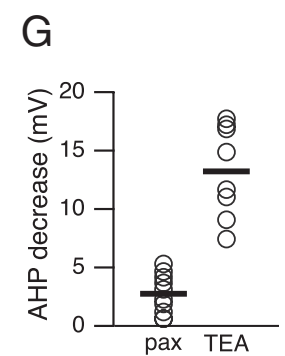

$I_{\mathrm{H}}$. Following the offset of the hyperpolarizing step, most neurons exhibited an increase in firing rate above baseline levels. The magnitude and time course of this rebound firing varied considerably across neurons, as evidenced by the examples in Figure 7, A1 and B1. A subset of neurons exhibited a transient decrease in firing rate following hyperpolarization offset; these neurons were concentrated in pontine nuclei and the NRTP.

Rebound firing responses were quantified across the population of precerebellar neurons both as the change in rate relative to baseline (Fig. 7C) (see Materials and Methods) and as the delay from the offset of hyperpolarization to the time of the next spike (Fig. 7D). Rebound responses differed quantitatively both within and across nuclei. The most pronounced rebound responses were observed in the external cuneate and MVN, in which several neurons fired transiently at 30 to 100 spikes per second above the baseline rate of 10 spikes per seconds (Fig. 7C). Neurons in both of these nuclei varied considerably, however, with many neurons exhibiting little or no rebound firing; such diversity mirrors that observed previously in MVN neurons (Sekirnjak and du Lac, 2002). In contrast, pontine neurons tended to exhibit little or no rebound firing (Fig. 7C). Furthermore, the time to the first spike after hyperpolarization offset was markedly longer in pontine neurons than in each of the other precerebellar nuclei (Fig. 7D). These results are consistent with the activation of an outward current that opposes postinhibitory rebound firing (Sekirnjak and du Lac, 2002), and they imply that the amplitudes or nature of the ionic currents near threshold vary considerably across precerebellar nuclei.

To examine mechanisms underlying rebound firing in precerebellar neurons, we pharmacologically blocked $I_{\mathrm{H}}$, the dominant inward current activated by neuronal hyperpolarization. Application of the ZD7288, an $I_{\mathrm{H}}$ blocker, reduced the depolarizing sag during steady hyperpolarization (Figs. 7A2,B2). Blockade of $I_{\mathrm{H}}$ also reduced the magnitude of rebound firing in the neurons that exhibited rebound firing in control conditions $(p<0.01$; Fig. $7 E)$, and it consistently increased the delay to the subsequent spike $(p<0.05$; Fig. $7 F)$. Interestingly, in the two neurons examined with exceptionally pronounced rebound firing (including the example in Fig. $7 B$ ), blockade of $I_{\mathrm{H}}$ had a relatively small influence on rebound firing. In summary, postinhibitory rebound firing varies considerably within and across precerebellar nuclei, suggesting differential expression of ionic conductances activated at membrane potentials around or below spike threshold.

\section{Discussion}

Precerebellar neurons are remarkably diverse with respect to the nature and sources of the signals that their mossy fiber axons convey to the cerebellum. To determine whether their intrinsic electrophysiological properties reflect this diversity, we subjected neurons recorded in eight different precerebellar nuclei in brainstem slices to a common battery of intracellular stimuli. All precerebellar mossy fiber neurons could transform inputs into 

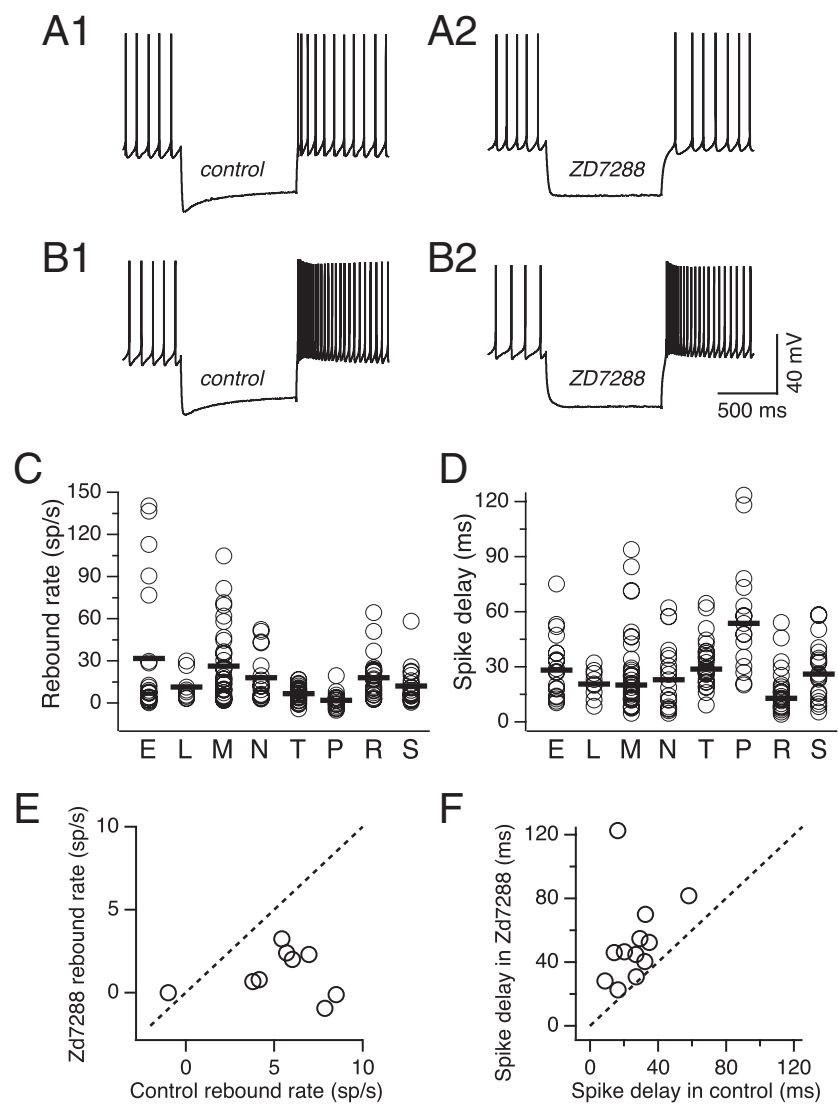

Figure 7. Postinhibitory rebound firing and the effect of $/_{H}$ blockade varies across and within precerebellar nuclei. $\boldsymbol{A 1}$, Example of firing responses to a 1 s hyperpolarization in a precerebellar neuron recorded in the lateral reticular nucleus. $\boldsymbol{A} \mathbf{2}$, Same neuron as in $\boldsymbol{A} \mathbf{1}$ after application of the $I_{H}$ blocker ZD7288 $(50 \mu \mathrm{M})$. B1, Firing responses to hyperpolarizing inputs in a second exemplar, recorded in the external cuneate. $\boldsymbol{B 2}$, Same neuron as in $\boldsymbol{A} \mathbf{1}$ after application of the $I_{\mathrm{H}}$ blocker ZD7288 $(50 \mu \mathrm{M})$. Neurons were maintained at baseline firing rates of 10 spikes per second with $D C$ current. $C$, Peak rebound firing rate (baseline subtracted) for all precerebellar neurons recorded. Significant differences $(p<0.05)$ are Pversus L, M, N, R, or S, and T versus R. $\boldsymbol{D}$, Delay from hyperpolarization offset to the next spike in all precerebellar neurons recorded. $\boldsymbol{E}$, Peak rebound firing rate after $I_{\mathrm{H}}$ blockade with ZD7288 is plotted versus the control rebound rate for precerebellar neurons recorded in the external cuneate nucleus $(n=3)$, nucleus of Roller $(n=5), \operatorname{NRTP}(n=2)$, and LRN and pontine nuclei $(n=1$ each). Two neurons in the external cuneate with rebound firing rates of $>50$ spikes per second are not shown. The diagonal line represents no change. $\boldsymbol{F}$, Delay to first posthyperpolarization spike after ZD7288 is plotted versus control delay for the same precerebellar neurons plotted in $\boldsymbol{E}$. E, External cuneate nucleus; L, lateral reticular nucleus; $\mathrm{M}$, medial vestibular nucleus; $\mathrm{N}$, nucleus prepositus hypoglossi; $T$, nucleus reticularis tegmenti pontis; $P$, pontine nucleus; $R$, nucleus of Roller/nucleus intercalatus; $S$, supragenual nucleus.

sustained, linear increases in firing rates over a wide range of rates. Pharmacological analyses revealed common expression of Kv3 and SK currents, which are critical for rapid firing and linearity, respectively. Precerebellar neurons differed within and across nuclei in their firing responses to the offset of hyperpolarizing currents, suggesting differential expression of subthreshold conductances. Together, these results indicate that precerebellar neurons perform similar scaling operations on excitatory inputs but may be differentially responsive to inhibitory inputs.

\section{Influence of intrinsic excitability on precerebellar neuronal firing responses in vivo}

When recorded in vivo, precerebellar neurons display strikingly different firing patterns that range from discrete, high-frequency bursts (Garwicz et al., 1998; Rancz et al., 2007; Bengtsson and
Jorntell, 2009) to graded responses (van Kan et al., 1993; Gamlin and Clarke, 1995; Cheron et al., 1996; Mackie et al., 1999; Arenz et al., 2008). Our findings demonstrate that all precerebellar neurons share the intrinsic capacity to sustain firing in proportion to their inputs over a wide range of firing rates. Classical studies employing intracellular recordings in vivo demonstrated similar broadband, linear spike generation in NRTP and in Clarke's column, a precerebellar spinal nucleus (Eide et al., 1969; Kitai et al., 1976). Together, these findings suggest that qualitative differences in firing responses observed in precerebellar neurons in vivo largely reflect differences in the duration and strength of synaptic inputs, rather than differentially tuned intrinsic excitability.

Although precerebellar neurons behave similarly in the firing domain, they differ substantially in their responses to hyperpolarizing inputs. Interestingly, neurons responsible for conveying sensory signals to the cerebellum (MVN, external cuneate) exhibited the largest postinhibitory rebound firing and tended to fire spontaneously at rest. At the other extreme, precerebellar neurons driven by cortical synapses (NRTP, pontine) exhibited little or no rebound firing and were silent at rest, consistent with previous findings in pontine neurons (Schwarz et al., 1997; Mock et al., 2002). These observations have functional implications for signal processing in different precerebellar circuits. Neurons that fire spontaneously and that have postinhibitory rebound could exploit synaptic inhibition to produce well-timed bursts of action potentials, such as those observed in the external cuneate nucleus in vivo (Mackie et al., 1999; Bengtsson and Jorntell, 2009). In contrast, intrinsic properties in non-rebounding neurons would favor integration of inhibitory and excitatory synaptic inputs.

\section{Mechanisms of fast, linear firing}

A small number of ionic mechanisms are critical for the ability of precerebellar neurons to respond to depolarizing stimuli with linear firing over a wide range. Firing at high rates requires adequate sodium channel availability during short interspike intervals. Neurons in precerebellar nuclei express Na currents that are specialized to minimize inactivation, including persistent (Schwarz et al., 1997; Gittis and du Lac, 2008) and resurgent currents (Gittis and du Lac, 2008; Gittis et al., 2010). These biophysical specializations are complemented by strong expression of Kv3 currents, which preclude Na channel inactivation by rapidly repolarizing the membrane potential (Lien and Jonas, 2003; Carter and Bean, 2009, 2011; Gittis et al., 2010) and are required for precerebellar neurons to sustain high firing rates (Fig. 6). A particularly important role for Kv3.3 channel subunits is suggested by prominent expression of Kv3.3 subunits in projection neurons in precerebellar nuclei, including the MVN (Brooke et al., 2010; Gittis et al., 2010), NPH (Kolkman et al., 2011), and pontine nuclei (Chang et al., 2007).

The wide input range over which precerebellar neurons can fire linearly depends critically on calcium-dependent $\mathrm{K}$ currents, which dampen excitability in the interspike interval. Our findings indicate that the calcium-dependent AHP identified previously in Clarke's column and pontine neurons (Gustafsson et al., 1978; Schwarz et al., 1997) is mediated predominantly by SK currents, with a minor contribution from BK currents. The calcium-dependence of SK currents and their delayed activation after the action potential (Smith et al., 2002) make them well suited for reducing excitability during sustained periods of firing while enabling transient high-frequency burst firing. Although the influence of channel blockers was examined in a small number of neurons, pharmacological effects were qualitatively consistent across neurons. Quantitative similarities in action potential 
waveforms, which reflect underlying ionic currents (Bean, 2007), suggest that our findings are applicable to all precerebellar neurons.

BK currents reliably influence gain in precerebellar neurons but appear to be dispensable for wide-range linear firing. What role might BK channels play in cerebellar signal processing? An intriguing possibility, suggested by studies of vestibular nucleus neurons, is that BK channels could regulate intrinsic gain in an activity-dependent fashion. In MVN neurons, repeated inhibition triggers persistent potentiation of intrinsic excitability via a CaMKII-dependent reduction of BK currents (Nelson et al., 2003, 2005). A similar mechanism, if expressed in precerebellar neurons that receive strong inhibition, could endow cerebellar circuits with a site of plasticity that controls the strength of mossy fiber signals.

\section{Functional significance for mossy fiber synaptic transmission in the cerebellar cortex}

Several properties of mossy fiber synapses enable precerebellar neurons to transmit both low- and high-frequency signals to their postsynaptic partners. Signals conveyed by low firing rates at the mossy fiber-to-granule cell synapse are enhanced via a large amount of glutamate spillover which contributes about half of the synaptic conductance (DiGregorio et al., 2002). The mossy fibergranule cell synapse is also under tonic inhibition from GABAergic Golgi cells, and modulation of this inhibition can regulate sensitivity to low-frequency inputs (Chadderton et al., 2004). Transmission of high-frequency signals is aided by fast vesicle reloading (Saviane and Silver, 2006; Hallermann et al., 2010) and desensitization-resistant AMPA receptors (DiGregorio et al., 2007).

Mossy fibers also synapse on Golgi and unipolar brush interneurons, which provide feedforward inhibition and excitation, respectively, to cerebellar granule cells. The mossy fiberGolgi cell synapse is strong, but activating mossy fibers beyond 50 spikes per second does not lead to an increase in Golgi cell firing (Kanichay and Silver, 2008). Therefore, high-frequency signals may be filtered at this synapse. Mossy fiber-unipolar brush synapses are specialized to entrap glutamate allowing for a prolonged glutamatergic signal lasting up to $5 \mathrm{~s}$ (Kinney et al., 1997). Little is known about the sensitivity of unipolar brush cells to presynaptic firing rate, but glutamate accumulation is unlikely to preserve the timing of mossy fiber input signals. Thus, linearly encoded inputs conveyed by mossy fiber axons are transformed in a nonlinear manner by feedforward inhibitory and excitatory circuits within the cerebellar cortex.

\section{Implications for development and signal processing in cerebellar circuits}

Precerebellar neurons share a common developmental origin. Born in the ventral portion of the rhombic lip, developing mossy fiber neurons express a common set of genes as they migrate along the rostral-caudal axis of the brain to their final locations in precerebellar nuclei (Rodriguez and Dymecki, 2000). It seems likely that a common transcriptional program is responsible for the shared intrinsic physiological properties of precerebellar neurons evidenced by our results. We speculate that differences in intrinsic spontaneous and rebound firing across functionally distinct precerebellar neurons could reflect activity-dependent regulation of ionic currents via sources of synaptic inhibition and/or excitation specific to each nucleus.

Linear signal processing at both the input and output stages may be critical for the cerebellum to function as an adaptive filter (Dean et al., 2010). The broad linear firing range, common ex- pression of Kv3 and SK currents, and differential expression of postinhibitory rebound currents in precerebellar neurons demonstrated in this study have also been observed in deep cerebellar nuclei (Jahnsen, 1986; Aizenman and Linden, 1999; Raman et al., 2000; Molineux et al., 2006; Uusisaari et al., 2007; Bagnall et al., 2009; Tadayonnejad et al., 2010) and in vestibular nuclei (Smith et al., 2002, Sekirnjak and du Lac, 2003; Gittis and du Lac, 2007). These neurons differ both intrinsically and computationally from from intensively studied pyramidal cells of the hippocampus and cortex. Our findings suggest that precerebellar mossy fiber neurons, together with deep cerebellar and vestibular neurons, can be considered members of a canonical class of neurons specialized for linear filtering over a wide dynamic range.

\section{References}

Aizenman CD, Linden DJ (1999) Regulation of the rebound depolarization and spontaneous firing patterns of deep nuclear neurons in slices of rat cerebellum. J Neurophysiol 82:1697-1709.

Arenz A, Silver RA, Schaefer AT, Margrie TW (2008) The contribution of single synapses to sensory representation in vivo. Science 321:977-980.

Bagnall MW, Stevens RJ, du Lac S (2007) Transgenic mouse lines subdivide medial vestibular nucleus neurons into discrete, neurochemically distinct populations. J Neurosci 27:2318-2330.

Bagnall MW, Zingg B, Sakatos A, Moghadam SH, Zeilhofer HU, du Lac S (2009) Glycinergic projection neurons of the cerebellum. J Neurosci 29:10104-10110.

Bean BP (2007) The action potential in mammalian central neurons. Nat Rev Neurosci 8:451-465.

Bengtsson F, Jorntell H (2009) Sensory transmission in cerebellar granule cells relies on similarly coded mossy fiber inputs. Proc Natl Acad Sci U S A 106:2389-2394.

Brooke RE, Corns L, Edwards IJ, Deuchars J (2010) Kv3.3 immunoreactivity in the vestibular nuclear complex of the rat with focus on the medial vestibular nucleus: targeting of Kv3.3 neurones by terminals positive for vesicular glutamate transporter 1. Brain Res 1345:45-58.

Carter BC, Bean BP (2009) Sodium entry during action potentials of mammalian neurons: incomplete inactivation and reduced metabolic efficiency in fast-spiking neurons. Neuron 64:898-909.

Carter BC, Bean BP (2011) Incomplete inactivation and rapid recovery of voltage-dependent sodium channels during high-frequency firing in cerebellar Purkinje neurons. J Neurophysiol 105:860-871.

Chadderton P, Margrie TW, Hausser M (2004) Integration of quanta in cerebellar granule cells during sensory processing. Nature 428:856-860.

Chang SY, Zagha E, Kwon ES, Ozaita A, Bobik M, Martone ME, Ellisman MH, Heintz N, Rudy B (2007) Distribution of Kv3.3 potassium channel subunits in distinct neuronal populations of mouse brain. J Comp Neurol 502:953-972.

Cheron G, Escudero M, Godaux E (1996) Discharge properties of brain stem neurons projecting to the flocculus in the alert cat. I. Medical vestibular nucleus. J Neurophysiol 76:1759-1774.

Dean P, Porrill J, Ekerot CF, Jorntell H (2010) The cerebellar microcircuit as an adaptive filter: experimental and computational evidence. Nat Rev Neurosci 11:30-43.

DiGregorio DA, Nusser Z, Silver RA (2002) Spillover of glutamate onto synaptic AMPA receptors enhances fast transmission at a cerebellar synapse. Neuron 35:521-533.

DiGregorio DA, Rothman JS, Nielsen TA, Silver RA (2007) Desensitization properties of AMPA receptors at the cerebellar mossy fiber granule cell synapse. J Neurosci 27:8344-8357.

Eccles JC, Faber DS, Murphy JT, Sabah NH, Taborikova H (1971) Afferent volleys in limb nerves influencing impulse discharges in cerebellar cortex. I. In mossy fibers and granule cells. Exp Brain Res 13:15-35.

Eide E, Fedina L, Jansen J, Lundberg A, Vyklicky L (1969) Properties of Clarke's column neurones. Acta Physiol Scand 77:125-144.

Escudero M, Cheron G, Godaux E (1996) Discharge properties of brain stem neurons projecting to the flocculus in the alert cat. II. Prepositus hypoglossal nucleus. J Neurophysiol 76:1775-1785.

Feng G, Mellor RH, Bernstein M, Keller-Peck C, Nguyen QT, Wallace M, Nerbonne JM, Lichtman JW, Sanes JR (2000) Imaging neuronal subsets in transgenic mice expressing multiple spectral variants of GFP. Neuron 28:41-51. 
Gamlin PD, Clarke RJ (1995) Single-unit activity in the primate nucleus reticularis tegmenti pontis related to vergence and ocular accommodation. J Neurophysiol 73:2115-2119.

Garwicz M, Jorntell H, Ekerot CF (1998) Cutaneous receptive fields and topography of mossy fibres and climbing fibres projecting to cat cerebellar C3 zone. J Physiol 512:277-293.

Gittis AH, du Lac S (2007) Firing properties of GABAergic versus nonGABAergic vestibular nucleus neurons conferred by a differential balance of potassium currents. J Neurophysiol 97:3986-3996.

Gittis AH, du Lac S (2008) Similar properties of transient, persistent, and resurgent $\mathrm{Na}$ currents in GABAergic and non-GABAergic vestibular nucleus neurons. J Neurophysiol 99:2060-2065.

Gittis AH, Moghadam SH, du Lac S (2010) Mechanisms of sustained high firing rates in two classes of vestibular nucleus neurons: differential contributions of resurgent $\mathrm{Na}, \mathrm{Kv} 3$, and BK currents. J Neurophysiol 104:1625-1634.

Gustafsson B, Linstrom S, Zangger P (1978) Firing behaviour of dorsal spinocerebellar tract neurones. J Physiol 275:321-343.

Hallermann S, Fejtova A, Schmidt H, Weyhersmuller A, Silver RA, Gundelfinger ED, Eilers J (2010) Bassoon speeds vesicle reloading at a central excitatory synapse. Neuron 68:710-723.

Jahnsen H (1986) Extracellular activation and membrane conductances of neurones in the guinea-pig deep cerebellar nuclei in vitro. J Physiol 372:149-168.

Joho RH, Hurlock EC (2009) The role of Kv3-type potassium channels in cerebellar physiology and behavior. Cerebellum 8:323-333.

Kanichay RT, Silver RA (2008) Synaptic and cellular properties of the feedforward inhibitory circuit within the input layer of the cerebellar cortex. J Neurosci 28:8955-8967.

Kinney GA, Overstreet LS, Slater NT (1997) Prolonged physiological entrapment of glutamate in the synaptic cleft of cerebellar unipolar brush cells. J Neurophysiol 78:1320-1333.

Kitai ST, Kocsis JD, Kiyohara T (1976) Electrophysiological properties of nucleus reticularis tegmenti pontis cells: antidromic and synaptic activation. Exp Brain Res 24:295-309.

Kolkman KE, Moghadam SH, du Lac S (2011) Intrinsic physiology of identified neurons in the prepositus hypoglossi and medial vestibular nuclei. J Vestib Res 21:33-47.

Lien CC, Jonas P (2003) Kv3 potassium conductance is necessary and kinetically optimized for high-frequency action potential generation in hippocampal interneurons. J Neurosci 23:2058-2068.

Lisberger SG, Fuchs AF (1978) Role of primate flocculus during rapid behavioral modification of vestibuloocular reflex. II. Mossy fiber firing patterns during horizontal head rotation and eye movement. J Neurophysiol 41:764-777.

Mackie PD, Morley JW, Rowe MJ (1999) Signalling of static and dynamic features of muscle spindle input by external cuneate neurones in the cat. J Physiol 519:559-569.

McKay BE, Turner RW (2004) Kv3 $\mathrm{K}^{+}$channels enable burst output in rat cerebellar Purkinje cells. Eur J Neurosci 20:729-739.

Mock M, Schwarz C, Thier P (2002) Serotonergic control of cerebellar mossy fiber activity by modulation of signal transfer by rat pontine nuclei neurons. J Neurophysiol 88:549-564.

Molineux ML, McRory JE, McKay BE, Hamid J, Mehaffey WH, Rehak R, Snutch TP, Zamponi GW, Turner RW (2006) Specific T-type calcium channel isoforms are associated with distinct burst phenotypes in deep cerebellar nuclear neurons. Proc Natl Acad Sci U S A 103:5555-5560.

Nelson AB, Krispel CM, Sekirnjak C, du Lac S (2003) Long-lasting increases in intrinsic excitability triggered by inhibition. Neuron 40:609-620.

Nelson AB, Gittis AH, du Lac S (2005) Decreases in CaMKII activity trigger persistent potentiation of intrinsic excitability in spontaneously firing vestibular nucleus neurons. Neuron 46:623-631.

Noda H (1986) Mossy fibres sending retinal-slip, eye, and head velocity signals to the flocculus of the monkey. J Physiol 379:39-60.
Oliva AA Jr, Jiang M, Lam T, Smith KL, Swann JW (2000) Novel hippocampal interneuronal subtypes identified using transgenic mice that express green fluorescent protein in GABAergic interneurons. J Neurosci 20:3354-3368.

Raman IM, Gustafson AE, Padgett D (2000) Ionic currents and spontaneous firing in neurons isolated from the cerebellar nuclei. J Neurosci 20:9004-9016.

Rancz EA, Ishikawa T, Duguid I, Chadderton P, Mahon S, Hausser M (2007) High-fidelity transmission of sensory information by single cerebellar mossy fibre boutons. Nature 450:1245-1248.

Rodriguez CI, Dymecki SM (2000) Origins of the precerebellar system. Neuron 27:475-486.

Rudy B, McBain CJ (2001) Kv3 channels: voltage-gated $\mathrm{K}^{+}$channels designed for high-frequency repetitive firing. Trends Neurosci 24:517-526.

Sah P (1996) $\mathrm{Ca}(2+)$-activated $\mathrm{K}+$ currents in neurones: types, physiological roles and modulation. Trends Neurosci 19:150-154.

Saviane, C Silver RA (2006) Fast vesicle reloading and a large pool sustain high bandwidth transmission at a central synapse. Nature 439:983-987.

Schwarz C, Mock M, Thier P (1997) Electrophysiological properties of rat pontine nuclei neurons in vitro. I. Membrane potentials and firing patterns. J Neurophysiol 78:3323-3337.

Sekirnjak C, du Lac S (2002) Intrinsic firing dynamics of vestibular nucleus neurons. J Neurosci 22:2083-2095.

Sekirnjak C, du Lac S (2006) Physiological and anatomical properties of mouse medial vestibular nucleus neurons projecting to the oculomotor nucleus. J Neurophysiol 95:3012-3023.

Sekirnjak C, Vissel B, Bollinger J, Faulstich M, du Lac S (2003) Purkinje cell synapses target physiologically unique brainstem neurons. J Neurosci 23:6392-6398.

Shin, M., Moghadam SH, Sekirnjak C, Bagnall MW, Kolkman, KE, Jacobs R, Faulstich M, du Lac, S. (2011) Multiple types of cerebellar target neurons and their circuitry in the vestibulo-ocular reflex. J Neurosci 31: 10776-10786.

Shino M, Ozawa S, Furuya N, Saito Y (2008) Membrane properties of excitatory and inhibitory neurons in the rat prepositus hypoglossi nucleus. Eur J Neurosci 27:2413-2424.

Smith MR, Nelson AB, du LacS (2002) Regulation of firing response gain by calcium-dependent mechanisms in vestibular nucleus neurons. J Neurophysiol 87:2031-2042.

Tadayonnejad R, Anderson D, Molineux ML, Mehaffey WH, Jayasuriya K, Turner RW (2010) Rebound discharge in deep cerebellar nuclear neurons in vitro. Cerebellum 9:352-374.

Takazawa T, Saito Y, Tsuzuki K, Ozawa S (2004) Membrane and firing properties of glutamatergic and GABAergic neurons in the rat medial vestibular nucleus. J Neurophysiol 92:3106-3120.

Tziridis K, Dicke PW, Thier P (2009) The role of the monkey dorsal pontine nuclei in goal-directed eye and hand movements. J Neurosci 29:6154-6166

Tziridis K, Dicke PW, Thier P (2011) Pontine reference frames for the sensory guidance of movement. Cereb Cortex. Advance online publication. doi:10.1093/cercor/bhr109.

Uusisaari M, Obata K, Knopfel T (2007) Morphological and electrophysiological properties of GABAergic and non-GABAergic cells in the deep cerebellar nuclei. J Neurophysiol 97:901-911.

van Kan PL, Gibson AR, Houk JC (1993) Movement-related inputs to intermediate cerebellum of the monkey. J Neurophysiol 69:74-94.

Zeilhofer HU, Studler B, Arabadzisz D, Schweizer C, Ahmadi S, Layh B, Bosl MR, Fritschy JM (2005) Glycinergic neurons expressing enhanced green fluorescent protein in bacterial artificial chromosome transgenic mice. J Comp Neurol 482:123-141.

Zhang Y, Partsalis AM, Highstein SM (1995) Properties of superior vestibular nucleus flocculus target neurons in the squirrel monkey. I. General properties in comparison with flocculus projecting neurons. J Neurophysiol 73:2261-2278. 